\title{
Safety and Efficacy of Single Intra-Articular Injection of a Cross-Linked Hyaluronic Acid/Mannitol Formulation [Happycross $®]$ in knee Osteoarthritis Results of a Prospective Observational Study in Daily Practice Conditions
}

\author{
Matthieu Monet, Ana-Maria Bozgan and Thierry Conrozier* \\ Department of Rheumatology, Hopital Nord Franche-Comté, France \\ Submission: March 16, 2017; Published: March 24, 2017
}

*Corresponding author: Thierry Conrozier, Department of rheumatology, Hôpital Nord Franche-Comté, 100 route de MOVAL, CS 10499 Trevenans, 90015 Belfort, France, Tel: +33 6869192 18; Email: thierry.conrozier@hnfc.fr; thierry_conrozier@hotmail.fr

\begin{abstract}
Objectives: To assess, in daily practice conditions, both safety and efficacy of a single intra-articular injection of a cross-linked hyaluronic acid (HA) combined with mannitol (HANOX-M-XL) in patients with symptomatic knee osteoarthritis.

Methods: We analyzed the clinical and radiological data of 53 consecutive patients with symptomatic knee OA, treated between January and June 2016 with a single intra-articular injection of HANOX-M-XL, a viscosupplement made of a cross-linked HA (16 mg/ml) + mannitol (35 mg/ $\mathrm{ml})$. The efficacy endpoints included the changes over time in the normalized (0-10) WOMAC pain, function and total and the patient's global assessment (PGA). Patient Acceptable Symptom State (PASS) and Minimum Clinically Important Improvement (MCII) were calculated from WOMAC pain sub-score. Patient's self assessment of treatment efficacy (0-3) and the decrease in analgesic consumption (0-5) were also obtained at the end of follow-up. Safety was assessed by recording all adverse events.
\end{abstract}

Results: During the selected period of time 53 patients with knee OA were treated with HANOX-M-XL. The mean follow-up duration was 28 weeks (18 to 32). No patient was lost to follow-up. At baseline the mean WOMAC pain sub-score and PGA were 4.6 (1.2) and 6.0 (1.1) respectively. The average decrease of WOMAC score over the 6 month follow-up was $>50 \%$, versus baseline values, for both pain and function $(\mathrm{P}<0.0001)$. At month $6,82 \%$ of patients were PASS + and $86.8 \%$ had experienced improvement $>$ MCII threshold. Three patients experienced local adverse events (increase of knee pain) after injection that resolved within 3 days.

Conclusion: In daily practice conditions, treatment with a single IA injection of HAnox-M-XL alleviates by more than $50 \%$ knee $0 \mathrm{~A}$ symptoms, over 6 months, in a large majority of patients, without safety concern.

Level of evidence: Knee osteoarthritis (OA) is a major cause of pain and disability in subjects over 50, whose incidence increases dramatically in western countries [1,2]. The medical management for knee OA includes a combination of nonpharmacological and pharmacological modalities [3-6]. Among the latter viscosupplementation with intra-articular (IA) injections of high molecular weight of hyaluronic acid (HA) has been suggested to be the most efficacious treatment, as evidenced by an effectsize of 0.63 [7]. However many controversies are still remaining regarding the clinical relevance and the contextual part of this effect [8-13].

Keywords: Hyaluronic acid; Viscosupplementation; Knee osteoarthritis; Mannitol; Intra-articular injection; Pilot study

Abbreviations: PGA: Patient's Global Assessment; MCII: Minimum Clinically Important Improvement; PASS: Patient Acceptable Symptom State

\section{Introduction}

Several authors underlined the possibility of differential effects between the many marketed HA medical devices [14-16]. Differences between products might be due to HA molecular weight, concentration or molecular organization (linear or cross-linked). The usually recommended dosing regimen varies

from one to five weekly injections. The reason of the need of repeat injections is due to the very short IA residence time of injected HA. It ranges from few days for linear HA [17], that must be injected 3 to 5 consecutive weeks, to few weeks $[18,19]$ for the solutions of cross-linked HA which allow a single injection regimen. 
HAnox-M-XL (Happy Cross $^{\circledR}$, LABRHA SAS, Lyon, France) is a viscosupplement, that combines cross-linked sodium hyaluronate and mannitol. Because oxidative stress is the main reason of HA depolymerization [20], mannitol, a polyol scavenging radical oxygen species (ROS), is claimed to increase the IA residence time of HA $[21,22]$. In vitro, mannitol has been demonstrated to protect HA against ROS-mediated degradation [21-23]. In an animal model of adjuvant-induced arthritis, mannitol has also exhibited anti-inflammatory and antiedematous effects [24]. Thus, the combination of mannitol with HA is expected improve the viscosupplement performances. Several clinical trials demonstrated the good ratio risk/benefit of HAnox-M-XL in hip [25,26] and ankle [27] OA. In a randomized double-blind placebo controlled trial, performed in 81 patients with knee OA, a single IA injection of $2.2 \mathrm{ml}$ of HAnox-M-XL was shown to reduce serum levels of Coll2-1, a specific marker of cartilage degradation [28]. The aim of the present work, was to obtain data from daily practice conditions, in a cohort of patients with knee OA, treated with IA HAnox-M-XL, and followed using a standardized procedure.

\section{Patients and methods}

\section{Analyzed data}

The present analyzed data were obtained from a cohort of patients referred to the department of rheumatology of the North Franche-Comté hospital (Belfort, France) for symptomatic knee OA between February and August 2016. The French Authorities recommend using viscosupplementation in patients suffering from knee OA, only if pain is not sufficiently relieved by analgesics, non-steroidal anti-inflammatory drugs (NSAIDs) and non-pharmacological modalities. In our rheumatology department all patients who require viscosupplementation are followed using the same procedure. At time of injection(s) and at each follow-up visit, whenever the time interval between the visits and whatever the viscosupplement used, a standardized questionnaire is administered before clinical examination, after patients had given agreement to the scientific and anonymous usage of the data collected during consultations.

The standardized questionnaires includes demographic and anthropometric data, prior and present treatments for knee OA, Western Ontario \& McMaster Universities Osteoarthritis Index (WOMAC index) [29] using a 11-box numerical rating scale (NRS, range $0-10)$ for each of the 24 items $(0-240)$, patient's global assessment (PGA) of knee pain (0-10 NRS). All values are normalized to scores with a $0-10$ range. The evaluation of the target knee X-rays, available at the time of injection, includes Kellgren-Lawrence grading scale [30]. The names and daily doses of concomitant therapies, analgesics, NSAIDs, symptomatic slow acting drugs for OA (SYSADOA) are reported into the medical file.

The present work, being a retrospective analysis of data obtained in daily practice conditions in a single rheumatology center, did not need the National Ethics Committee approval. The procedure of follow-up (i.e. questionnaire, agreement form, statistics) was approved by the Hôpital Nord Franche-Comté scientific and ethics committee.

Treatment consisted in a single injection of HAnox-M-XL, a marketed viscosupplement made of $35.2 \mathrm{mg}$ of cross-linked HA and $77 \mathrm{mg}$ of mannitol, giving the gel a very high viscosity (i.e. $2560 \mathrm{~Pa} . \mathrm{s}$ at $0.01^{\mathrm{s}-1}$ ) [31]. The content of a $2.2 \mathrm{ml}$ syringe was injected into the target knee using a 21-gauge needle, after removal of synovial fluid effusion if present, using a lateral midpatellar approach, by experienced rheumatologists.

\section{Efficacy outcome measures}

The primary efficacy criterion was the decrease throughout the follow-up for normalized WOMAC pain sub-score (0-10). Other efficacy criteria were the absolute and relative changes in normalized WOMAC total and function scores, and PGA, between injection and month 6. Patient Acceptable Symptom State (PASS) and Minimum Clinically Important Improvement (MCII) [32] were calculated from WOMAC pain sub-score. At end-point, patients were also asked to self-assess the treatment efficacy $(0=$ null, $1=$ mild, 2 = good, 3 =very good). The decrease in analgesic/ NSAIDs intake was also self-assessed by the patient using a 5 point scale $(0 ; \leq 25 \%$; 26-50\%, 51-75\%; > 75\%).

\section{Safety evaluation}

The follow-up procedure planned to record adverse events (AEs) in accordance with the European standard EN ISO 14155: 2011. A particular care was applied for AEs occurring immediately and the very next days after injection.

\section{Statistics}

The XLstats ${ }^{\circledR} 2015$ (Addinsoft) software was used for carrying out the statistical analyses. A descriptive analysis was performed on all the collected data. Qualitative variables were described using frequencies and percentages. Quantitative variables were describes using mean, standard deviation (SD) and distribution (minimum, maximum and median). PASS and MCII were calculated from WOMAC pain sub-score absolute values at end-point and variations respectively. Predictive factors of response were searched using univariate comparison (chi-2 or Fischer's exact test, t-test or Mann and Whitney) regarding demographic and clinical characteristics of the patients.

\section{Results}

Fifty-three patients, 35 women and 18 men, received one injection of HAnox-M-XL for painful knee OA during the selected period of time. Full data were available in all subjects with an average follow-up duration of 28 weeks (range 18 to 32). Mean (SD) age was 62.6 (12.3) years and BMI $27.5(5.2) \mathrm{kg} / \mathrm{m}^{2}$. The average time from $\mathrm{OA}$ diagnosis and HAnox-M-XL injection was 54.0 (36.5) months. Knee OA was a bilateral in 35 patients (66\%). Kellgren- Lawrence grade was I, II, III and IV in 2, 18, 23 and 10 cases respectively. Fifty patients (94.3\%) were regular analgesics or NSAIDs consumers. Twenty-eight (52.8\%) subjects were treated with SYSADOA. Thirty-three (62.2\%) and 35 (66\%) 
patients have previously been treated with IA corticosteroid and/or HA, respectively. At time of injection the normalized WOMAC pain sub-score was 4.6 (1.2) ranging from 2.6 to 7.2 . The average normalized WOMAC function and total scores were both 3.9 (1.2). Mean PGA was 6.0 (1.1). Throughout the 6month follow-up, WOMAC pain, function and total scores decreased by $58 \%, 51.2 \%$ and $52.5 \%$ respectively (all $P=0.0001$ ) (Figure 1).

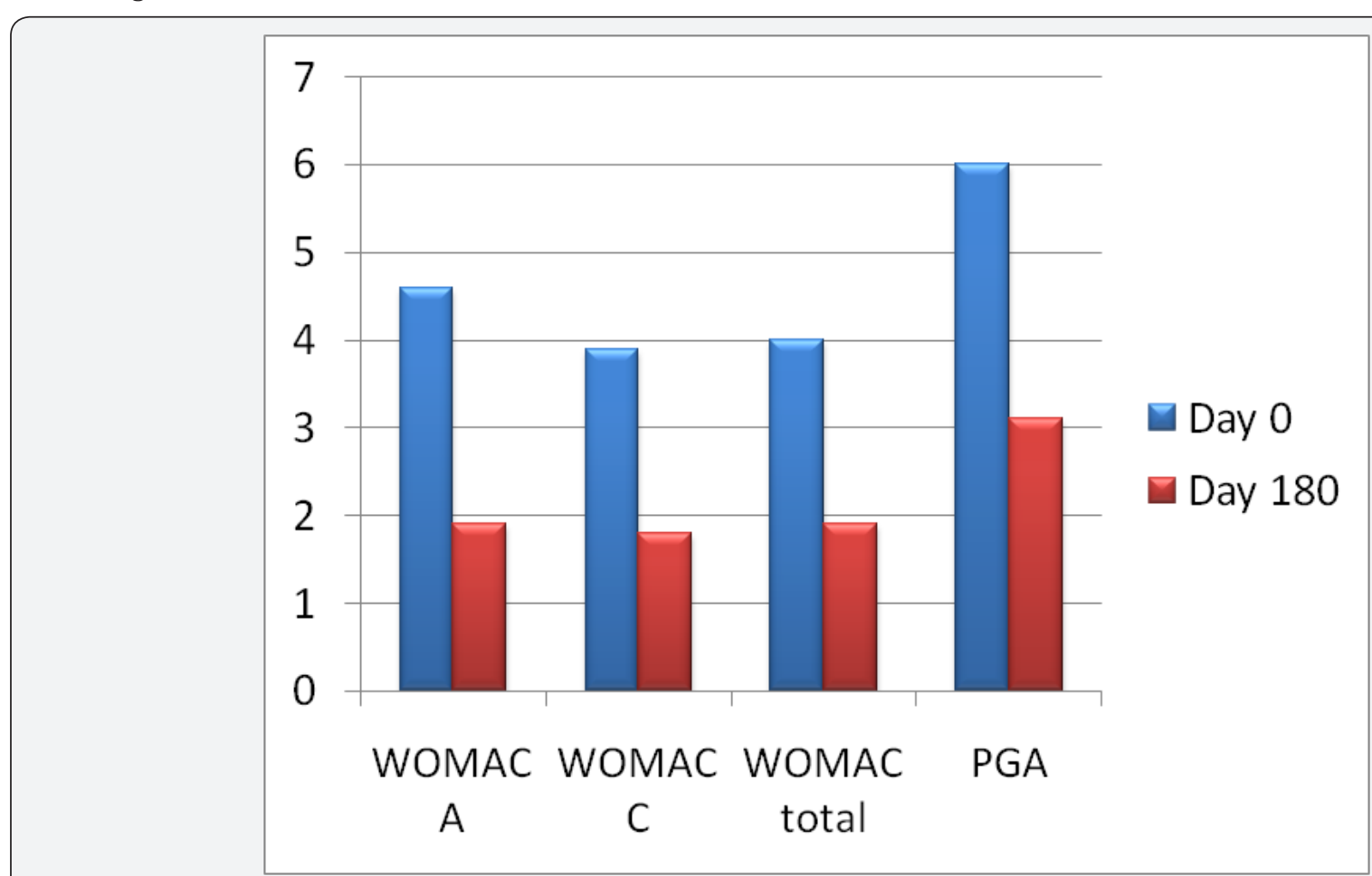

WOMAC: Western Ontario \& McMaster Universities Osteoarthritis Index normalized on a 11 point rating numeric scale (0-10)

WOMAC A: WOMAC pain sub-score normalized on a 11 point rating numeric scale (0-10)

WOMAC C: WOMAC function sub-score normalized on a 11 point rating numeric scale (0-10);

PGA: Patient global assessment of pain on a 11 point rating numeric scale (0-10);

Figure 1: Decrease over time of WOMAC A, WOMAC C, WOMAC total and PGA in 53 patients with knee OA treated with a single injection of HANOX-M-XL.

The mean PGA variation was $-48.5 \%$. All outcome variations were highly correlated between themselves (all $\mathrm{P}<0.0001$ ). At end-point efficacy was self-rated by patients as "good or very good" by $77.3 \%$ of patients. Eighty-two percent fulfilled the PASS and $86.8 \%$ the MCII criteria. The decrease of WOMAC pain over time was not statistically correlated with age, gender, BMI, $\mathrm{X}$-ray grade and disease duration (all $\mathrm{P}>0.05$ ). However when comparing patients with Kellgren grade I-II versus Kellgren grade III-IV, $\mathrm{P}$ value was close to the statistical significance $(\mathrm{P}=$ 0.07). Similarly there was a trend for a negative relationship between BMI and the decrease in WOMAC function $(\mathrm{P}=0.10)$ and total $(\mathrm{P}=0.06)$. This trend was confirmed by the percentage of obese patients $(\mathrm{BMI}>30)$ reaching the PASS threshold that was significantly lower than that of non-obese subjects $(\mathrm{P}=0.03)$. A decrease in analgesics/NSAIDs intake was reported by $86 \%$ of patients. By the end of the study, $60 \%$ of patients had reduced their intake by half or more. Regarding the safety, 3 target knee AEs were reported (5.7\%). All were characterized by transient worsening of pain occurring a few hours after injection. All resolved within 36 and 72 hours without other treatment than usually analgesics. There was no occurrence of joint effusion, no systemic $\mathrm{AE}$ and no severe $\mathrm{AE}$ related to treatment.

\section{Discussion}

The main relevance of the present work is that it has been achieved from daily practice by using the same tools of evaluation than that of interventional trials, giving the study a greater scientific value than that of a simple observational study. Our results showed that a single injection of HAnox-M$\mathrm{XL}$ alleviates pain and improves joint function significantly, over at least 6 months, in a wide majority of patients, without safety concern. Indeed, the present data confirmed that local and systemic tolerability of HAnox-M-XL was similar to that of regular viscosupplement not containing mannitol. These results were consistent with those of previous studies showing a good tolerability of viscosupplement combined with mannitol [25$28,33]$ or its isomer, sorbitol $[34,35]$.

Our study also highlights the fact that a single injection of HAnox-M-XL allows to reduce the use of analgesics by half or more in a majority of patients. In economic terms, this is an important point to take into account when assessing the cost effectiveness of viscosupplementation, seeing the direct cost of painkillers, and the indirect costs related to the potential problems of intolerance, common in an elderly population [36]. 
Our study suffers from several limitations. In real life conditions, patients were allowed to take NSAIDs, analgesics, SYSADOA and non-pharmacological therapies for OA.

We are not able to precisely assess their influence on pain and function changes. However as the study showed that a majority of patients decreased dramatically their intake of painkillers, their influence on the clinical results seems to be limited. The second limitation is the relatively small sample size that may explain the lack of statistically significant relationship between radiographic features and clinical response that has been demonstrated recently in 166 patients with knee OA [37]. Another limitation is the relative homogeneity of the patients' sample. Indeed patients with very severe disease, those with major risk factors of treatment failure (i.e. patients with both Kellgren grade IV and obesity, with large knee effusion or major joint malalignment, or those waiting for knee replacement) and those with failure of a previous viscosupplementation, were not eligible for viscosupplementation. This may explain the relatively low level of pain and disability scores at baseline as well as the high percentage of patients who reached the PASS definition.

In summary, in daily practice conditions, treatment of symptomatic knee OA with a single IA injection of HAnox-M-XL allows to alleviate pain and disability by more than $50 \%$ and to dramatically decrease painkillers consumption, in a large majority of patients, over a minimum period of time of 6 months, without safety concern.

\section{Disclosure of Interest in Relation with this Work}

Thierry Conrozier: Received honorarium from Labrha for expert and consultant services

\section{Ana-Maria Bozgan: None}

\section{Matthieu Monet: None}

\section{References}

1. Suri P, Morgenroth DC, Hunter DJ (2012) Epidemiology of osteoarthritis and associated comorbidities. PM R 4(5 Suppl): S9-S10.

2. Woolf AD, Pfleger B (2003) Burden of major musculoskeletal conditions. Bull World Health Organ 81(9): 646-656.

3. Jordan KM, Arden NK, Doherty M, Bannwarth B, Bijlsma JW, et al. (2003) EULAR Recommendations 2003: an evidence based approach to the management of knee osteoarthritis: Report of a Task Force of the Standing Committee for International Clinical Studies Including Therapeutic Trials [ESCISIT]. Ann Rheum Dis 62(12):1145-1155.

4. Hochberg MC, Altman RD, April KT, Benkhalti M, Guyatt G, et al. (2012) American College of Rheumatology 2012 recommendations for the use of nonpharmacologic and pharmacologic therapies in osteoarthritis of the hand, hip, and knee. Arthritis Care Res 64(4): 465-474.

5. McAlindon TE, Bannuru RR, Sullivan MC, Arden NK, Berenbaum F, M et al. (2014) OARSI guidelines for the non-surgical management of knee osteoarthritis. Osteoarthritis Cartilage 22(3): 363-388.

6. Bruyère $\mathrm{O}$, Cooper $\mathrm{C}$, Pelletier JP, Branco J, Luisa Brandi M, et al. (2014) An algorithm recommendation for the management of knee osteoarthritis in Europe and internationally: A report from a task force of the European Society for Clinical and Economic Aspects of Osteoporosis and Osteoarthritis [ESCEO]. Semin Arthritis Rheum 44(3): 253-263.

7. Bannuru RR, Schmid CH, Kent DM, Vaysbrot EE, Wong JB, et al. (2015) Comparative effectiveness of pharmacologic interventions for knee osteoarthritis: a systematic review and network meta-analysis. Ann Intern Med 162: 46-54.

8. Rutjes AW, Jüni P, da Costa BR, Trelle S, Nüesch E, et al. (2012) Viscosupplementation for osteoarthritis of the knee: a systematic review and meta-analysis. Ann Intern Med 157(3):180-191.

9. Jevsevar D, Donnelly P, Brown GA, Cummins DS (2015) Viscosupplementation for Osteoarthritis of the Knee: A Systematic Review of the Evidence. J Bone Joint Surg Am 97(24): 2047-2060.

10. Campbell KA, Erickson BJ, Saltzman BM, Mascarenhas R, Bach BR Jr, et al. (2015) Is Local Viscosupplementation Injection Clinically Superior to Other Therapies in the Treatment of Osteoarthritis of the Knee: A Systematic Review of Overlapping Meta-analyses. Arthroscopy 31(10): 2036-2045.

11. Cooper C, Rannou F, Richette P, Bruyère O, Al-Daghri N, et al. (2017) Use of Intra-Articular Hyaluronic Acid in the Management of Knee Osteoarthritis in Clinical Practice. Arthritis Care Res (Hoboken).

12. Miller LE, Block JE (2013) US-approved Intra-articular hyaluronic acid injections are safe and effective in patients with knee osteoarthritis: Systematic review and meta-analysis of randomized saline-controlled trials. Clin Med Insights Arthritis Musculoskelet Disord 6: 57-63.

13. Zou K, Wong J, Abdullah N, Chen X, Smith T, et al. (2016) Examination of overall treatment effect and the proportion attributable to contextual effect in osteoarthritis: meta-analysis of randomised controlled trials. Ann Rheum Dis 75(11):1964-1970.

14. Bellamy N, Campbell J, Robinson V, Gee T, Bourne R, et al. (2006) Viscosupplementation for the treatment of osteoarthritis of the knee. The Cochrane Database of Systematic Reviews 2.

15. Raman R, Henrotin Y, Chevalier X, Migliore A, Jerosch J, et al. (2017) Decision algorithms for the retreatment with viscosupplementation in patients suffering from knee osteoarthritis. recommendations from the European Viscosupplementation Consensus group (EUROVISCO). Cartilage.

16. Altman RD, Bedi A, Karlsson J, Sancheti P, Schemitsch E (2016) Product Differences in Intra-articular Hyaluronic Acids for Osteoarthritis of the Knee. Am J Sports Med 44(8): 2158-2165.

17. Lindenhayn K, Heilmann HH, Niederhausen T, Walther HU, Pohlenz K (1997) Elimination of tritium-labelled hyaluronic acid from normal and osteoarthritic rabbit knee joints. Eur J Clin Chem Clin Biochem 35(5): 355-363.

18. Lindqvist U, Tolmachev V, Kairemo K, Aström G, Jonsson E, et al. (2002) Elimination of stabilised hyaluronan from the knee joint in healthy men. Clin Pharmacokinet 41(8): 603-613.

19. Larsen NE, Dursema HD, Pollak CT, Skrabut EM (2011) Clearance kinetics of a hylan-based viscosupplement after intra-articular and intravenous administration in animal models. J Biomed Mater Res B Appl Biomater 100(2): 457-462.

20. Stern R, Kogan G, Jedrzejas MJ, Soltés L (2007) The many ways to cleave hyaluronan. Biotechnol Adv 25 (6): 537-557.

21. Conrozier T, Mathieu P, Rinaudo M (2014) Mannitol allows to preserve the elasto-viscous properties of hyaluronic acid in an in vitro model of oxidative stress. Rheumatology and Therapy 1: 45-54.

22. Rinaudo M, Lardy B, Grange L, Conrozier T (2014) Effect of mannitol on hyaluronic acid stability in two in vitro models of oxidative stress. Polymers 6(7): 1948-1957. 
23. Mendoza G, Alvarez AI, Pulido MM, Molina AJ, Merino G, et al. (2007) Inhibitory effects of different antioxidants on hyaluronan depolymerization. Carbohydr Res 342: 96-102.

24. Cavone L, Calosi L, Cinci L, Moroni F, Chiarugi A (2012) Topical mannitol reduces inflammatory edema in a rat model of arthritis. Pharmacology 89: 18-21.

25. Conrozier T, Bossert M, Balblanc JC, Sondag M, Walliser-Lohse A (2014) Viscosupplementation with HAnox-M-XL is effective in moderate hip osteoarthritis but is not an alternative to hip joint surgery in patients with severe disease. results of a clinical survey in 191 patients treated in daily practice. EMJD 3: 49-55.

26. Eymard F, Maillet B, Lellouche H, Mellac-Ducamp S, Brocq O, et al. (2017) Predictors of response to viscosupplementation in patients with hip osteoarthritis: results of a prospective, observational, multicentre, open-label, pilot study. BMC Musculoskelet Disord 18(1): 3.

27. Bossert M, Boublil D, Parisaux JM, Bozgan AM, Richelme E, et al. (2016) Imaging Guidance Improves the Results of Viscosupplementation with HANOX-M-XL in Patients with Ankle Osteoarthritis: Results of a Clinical Survey in 50 Patients Treated in Daily Practice. Clin Med Insights Arthritis Musculoskelet Disord 9: 195-199.

28. Henrotin Y, Berenbaum F, Chevalier X, Marty M, Richette P, et al. (2015) Significant reduction of the Serum Levels of a Specific Biomarker of Cartilage Degradation (serum Coll2-1) following Viscosupplementation Compared to Placebo in patients with Knee Osteoarthritis: the EPIKART Study. Topics di Terapia Intra-Articolare $7(2): 21-22$.

29. Bellamy N, Buchanan WW, Goldsmith CH, Campbell J, Stitt LW (1988) Validation study of WOMAC: a health status instrument for measuring clinically important patient relevant outcomes to antirheumatic drug therapy in patients with osteoarthritis of the hip or knee. J Rheumatol 15(12): 1833-1840.

30. Kellgren JH, Lawrence JS (1957) Radiological assessment of osteoarthrosis. Ann Rheum Dis 16(4): 494-502.

31. Conrozier T, Patarin J, Mathieu P, Rinaudo M (2016) Steroids, lidocain and ioxaglic acid modify the viscosity of hyaluronic acid: in vitro study and clinical implications. Springerplus 5: 170.

32. Bellamy N, Hochberg M, Tubach F, Martin-Mola E, Awada H, et al. (2015) Development of multinational definitions of minimal clinically important improvement and patient acceptable symptomatic state in osteoarthritis. Arthritis Care Res [Hoboken] 67(7): 972-980.

33. Borras-Verdera A, Calcedo-Bernal V, Ojeda-Levenfeld J, Clavel-Sainz C (2012) Efficacy and safety of a single intra-articular injection of $2 \%$ hyaluronic acid plus mannitol in knee osteoarthritis over a 6-month period. Rev Esp Cir Ortop Traumatol 56(4): 274-280.

34. Heisel J, Kipshoven C (2013) Safety and efficacy findings from a noninterventional study of a new hyaluronic acid/sorbitol formulation (Go-on $®$ Matrix) for intra-articular injection to relieve pain and disability in osteoarthritis patients. Drug Res 63(9): 445-449.

35. Migliore A, Massafra U, Bizzi E, Tormenta S, Cassol M, et al. (2014) Duration of symptom relief after intra-articular injection of hyaluronic acid combined with sorbitol (anti-ox-vs) in symptomatic hip osteoarthritis. Int J Immunopathol Pharmacol 27(2): 245-252.

36. Lanas A, Tornero J, Zamorano JL (2010) Assessment of gastrointestinal and cardiovascular risk in patients with osteoarthritis who require NSAIDs: the LOGICA study. Ann Rheum Dis 69(8): 1453-1458.

37. Eymard F, Chevalier X, Conrozier T (2017) Obesity and radiological severity are associated with viscosupplementation failure in patients with knee osteoarthritis. J Orthop Res.

\section{Your next submission with Juniper Publishers will reach you the below assets}

- Quality Editorial service

- Swift Peer Review

- Reprints availability

- E-prints Service

- Manuscript Podcast for convenient understanding

- Global attainment for your research

- Manuscript accessibility in different formats

(Pdf, E-pub, Full Text, Audio)

- Unceasing customer service

Track the below URL for one-step submission https://juniperpublishers.com/online-submission.php 